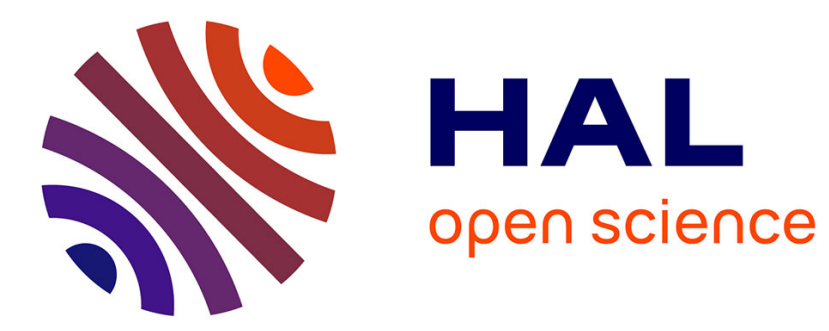

\title{
Prise en compte du spirituel et nouvelles représentations du soin \\ Tanguy Chatel
}

\section{To cite this version:}

Tanguy Chatel. Prise en compte du spirituel et nouvelles représentations du soin. Prise en compte du spirituel et nouvelles représentations du soin, Jun 2006, Grenoble, France. pp.196-200. halshs00311090

\section{HAL Id: halshs-00311090 \\ https://shs.hal.science/halshs-00311090}

Submitted on 12 Aug 2008

HAL is a multi-disciplinary open access archive for the deposit and dissemination of scientific research documents, whether they are published or not. The documents may come from teaching and research institutions in France or abroad, or from public or private research centers.
L'archive ouverte pluridisciplinaire HAL, est destinée au dépôt et à la diffusion de documents scientifiques de niveau recherche, publiés ou non, émanant des établissements d'enseignement et de recherche français ou étrangers, des laboratoires publics ou privés. 


\title{
"Prise en compte du spirituel et nouvelles représentations du soin »
}

\author{
Par Tanguy CHÂTEL, \\ Sociologue des religions (Ecole Pratique des Hautes Etudes, Paris) \\ Accompagnant bénévole (ASP Fondatrice, Paris) \\ Conférence donnée au congrès 2007 de la Société Française d'Accompagnement et de \\ soins Palliatifs (SFAP) à Grenoble. \\ Propos repris et publiés dans la revue Médecine palliative, $n^{\circ} 6$, juin 2007, p196-200.
}

«Leur nom révèle le projet de ces philosophes, on les appelle thérapeutes, d’abord parce que la médecine [iatrikè] dont ils font profession est supérieure à celle qui a cours dans nos cités, celle-ci ne soigne que les corps mais l'autre soigne aussi le psychisme en proie à ces maladies pénibles et difficiles à guérir...Pour le thérapeute, le corps ne peut pas seulement être considéré comme un objet, comme une chose ou une machine au fonctionnement défectueux quil s'agirait de "réparer »; le corps est un corps " animé ». Il n'y a pas de corps sans âme, ce qui n'a plus d'âme, n'étant plus animé, ne mérite pas le nom de corps mais de cadavre. " Philon d'Alexandrie, $\mathbf{1}^{\mathrm{er}}$ siècle après J.C ${ }^{1}$.

Le titre de cette table-ronde est doublement révélateur. Il fait d'abord clairement référence à la conception traditionnelle de la souffrance globale selon $\mathbf{C}$. Saunders ("total pain») et ses quatre composantes essentielles (physique, psychologique, sociale et spirituelle), qui demeure encore le principal outil théorique en matière de soin (global) à la personne. Mais il reflète également que cette représentation conserve ses propres espaces d'incertitude et d'insatisfaction, notamment autour de la manière de considérer la dimension spirituelle de l'être humain dans le processus de soin.

Après plusieurs années de recherches sur ce sujet, j'ai acquis la conviction que la souffrance spirituelle n'est pas seulement, comme on l'a longtemps cru, un aspect de la souffrance globale. Il ne s'agit pas d'une question particulière, référée à une compétence également particulière; il s'agit, au contraire, d'une question centrale touchant à la nature même de l'accompagnement. Ce qui se joue donc aujourd'hui autour de cette question si subtile et en même temps si complexe du spirituel, c'est, me semble-t-il, de faire évoluer en profondeur nos représentations en matière de souffrance et donc de faire évoluer, à la fois subtilement et audacieusement, nos pratiques de l'accompagnement.

Dans la pratique, la notion de total pain (qui peut aussi s'entendre comme la « somme des souffrances") a induit un certain morcellement de la souffrance par déplacement du regard du malade vers les professionnels de sa prise en charge : pour chaque type de souffrance est identifié un spécialiste et c'est par la juxtaposition combinée des spécialistes, réunis en équipe pluridisciplinaire, qu'on prétend aujourd’hui

LELOUP Jean-Yves, Prendre soin de l'être, Philon et les thérapeutes d'Alexandrie, coll. spiritualités vivantes, Albin Michel, 1999. 


\section{"Prise en compte du spirituel et nouvelles représentations du soin »}

le mieux répondre à la souffrance globale. L'attention tend à se porter davantage sur tel ou tel aspect plutôt que sur la souffrance réellement globale qui reste assez mal appréhendée. Peut-on se satisfaire de considérer que le spirituel est une dimension parmi d'autres de l'homme, qui se situerait à côté de ses autres dimensions physique, psychologique et sociale et au même titre qu'elles ? Peut-il suffire de considérer qu'elle est du seul ressort de spécialistes (essentiellement les ministres du culte) ? C'est cette conception qui, sur fond de laïcité complexée et prudente, a pourtant prévalu jusqu'à aujourd'hui. Or, notre société s'est, en peu de décennies considérablement sécularisée et laïcisée, avec une accélération formidable ces dernières années, et on peut voir aujourd'hui en abondance du spirituel qui ne se confond plus avec le religieux, auquel il a été totalement assimilé pendant des siècles ${ }^{2}$. Cet affranchissement du spirituel par rapport au religieux vient alors bousculer cette représentation de la souffrance globale car il n'y trouve plus bien sa place.

Il nous faut à présent oser aller au-delà des considérations trop générales sur la dimension spirituelle de l'homme qui réjouissent le poète ou le philosophe mais servent peu le praticien, et se donner les moyens d'y voir autrement clair, afin de se doter d'outils plus fins pour une pratique plus juste. C'est notre engagement de répondre au plus près au besoin des malades. Encore faut-il considérer ces besoins de leur point de vue de malade et non du notre de « spécialiste » de tel ou tel aspect de sa souffrance.

Ce thème du spirituel dans les soins - et le congrès 2007 de la SFAP dans sa problématique d'ensemble - signe donc le point de départ d'un cheminement conceptuel, ouvrant de nouvelles perspectives à la pratique du soin. Il peut donc, presque sans surprise, nous ramener à l'esprit initial des soins palliatifs pour leur donner une nouvelle vigueur et une autre profondeur.

\section{Spirituel, souffrance spirituelle et accompagnement de la souffrance spirituelle?}

Très rapidement, quelques éléments de cadrage puisque ces travaux ont déjà été publiés et qu'on pourra s'y référer pour davantage de précisions3.

1 - Tous les accompagnants se sentent plus ou moins concernés par la souffrance spirituelle. Mais non seulement ils peinent à l'identifier (s'agissant d'une souffrance rarement explicite), mais quand ils croient y parvenir, ils peinent aussi à l'accompagner, ne se sentant pas toujours légitimes à répondre à la demande d'un malade qui les choisit pourtant pour s'ouvrir à eux de sa souffrance dans ce domaine.

2 - Cela tient à une confusion culturelle et historique entre les notions de spirituel et de religieux qui conduit à saisir le ministre du culte de toute question supposée d'ordre entreprise, entre 2004 et 2006, par l'ASP de Paris, puis I'Union nationale des ASP (UNASP), sensibilisées à l'importance de cette question, mais aussi à l'imprécision régnant autour d'elle. Répondant aux demandes de bénévoles en demande d'éclairages pour leur pratique de l'accompagnement, ces associations ont entrepris de réunir des groupes bénévoles sur ce sujet sensible, qui ont confronté ces éléments de réflexion à leur pratique pour en valider la pertinence. Ces travaux ont ensuite fait l'objet d'un séminaire de formation interne, puis d'une présentation complète lors du congrès annuel de l'UNASP et enfin d'un article complet dans la revue Liaisons de juin 2006 auquel on se réfèrera pour plus d'informations. 


\section{"Prise en compte du spirituel et nouvelles représentations du soin »}

spirituel. Or, «il est clair qu'on peut parler ensemble du spirituel sans prendre les mots ou les rites d'une religion. " reconnaît le Père de Villers 4 . Il est donc essentiel de faire l'effort de distinguer le spirituel du religieux pour mieux comprendre les besoins réels du malade et l'accompagner de manière appropriée.

On peut déjà s'aider du vocabulaire courant pour mettre en évidence que " spirituel » et « religieux » ne sont pas exactement synonymes. Si parler de pratique religieuse ou de pratique spirituelle $\mathrm{a}$, à peu de choses près, le même sens, en revanche, il n'en va pas de même avec le mot « vie » qui s'emploie différemment, soit pour qualifier l'existence de personnes qui se sont consacrées à une mission contemplative ou apostolique ("vie religieuse »), soit pour décrire la vie intérieure propre à chacun qu’il soit religieux ou laïc («vie spirituelle»). Autre exemple, on parle aisément d'appartenance religieuse pour signifier une adhésion à un groupe ; à l'inverse, on ne saurait parler d' "appartenance spirituelle »5. Pour sa part, le terme " croyance » ressortit lui aussi essentiellement au domaine religieux mais traduit déjà un léger glissement hors de ce champ, vers quelque chose de plus spirituel ${ }^{6}$. A l'opposé, le terme " expérience " ne semble pouvoir s'accorder qu'avec l'adjectif "spirituel » puisque l'expression « expérience religieuse " n'a pas véritablement de sens dans le langage commun.

Ces premiers éléments posés, on peut poursuivre en distinguant :

- Ce qui relève du culte et des croyances (dimension rituelle), qui est de l'ordre de l'option personnelle, et qui se réfère à une religion en particulier, reposant sur une science composée de connaissances et de pratiques propres (préceptes, sacrements...). L'accompagnement dans ce registre relève alors spécifiquement des ministres du culte et de leurs représentants dûment formés au discours théologique et investis d'une autorité ;

- Et ce qui, indépendamment de tout culte ou croyances particulières, touche l'être humain en tant que tel (ontologiquement), dans sa dimension spirituelle, subtile et délicate, et pas nécessairement formelle. L'accompagnement repose alors plus sur une conscience que sur une science, plus sur un état d'être et une présence que sur un savoir(-faire), et relève alors plus largement de la responsabilité de tout être humain sensible et bienveillant.

Pour clarifier davantage, on peut encore affirmer, en forme de repère, le double principe suivant :

- Tout être humain a une dimension spirituelle. Il peut aussi choisir ou non d'avoir une religion.

- On peut vivre sa religion de manière spirituelle. On peut également la vivre de manière non spirituelle.

Il peut donc y avoir du religieux sans spirituel et réciproquement. Il ne s'agit certainement pas de les opposer dans une lecture qui serait alors caricaturale et probablement partisane. Il s'agit simplement de comprendre, malgré nos conditionnements historiques, que ces deux notions se distinguent au fond, quoiqu'elles puissent aussi heureusement se combiner. On peut donc dire que, par principe, il y a du

Malgré la nuit, Desclée de Brouwer, 1998.

5 Encore qu'on puisse parler de « famille spirituelle » pour signifier l'adhésion à un courant de pensée informel. Ce qui ne saurait revêtir le même sens communautaire fort.

6 Ce que la sociologue des religions Grace Davie met en évidence quand elle parle de « believing without belonging» : croire sans appartenir. 


\section{"Prise en compte du spirituel et nouvelles représentations du soin »}

spirituel dans la religion mais que la religion n'est pas le seul vecteur du spirituel. Philosophie, psychologie, nouvelles spiritualités, morale, sacré, symbolisation, magie, humanisme...et même athéisme7 sont autant de voies qui peuvent se prétendre, à différents égards, également porteuses de spirituel. Le spirituel semble donc être plus de l'ordre du générique que du particulier, du fond que de la forme, de l'individuel que du collectif, de l'expérience que de la croyance.

3 - Accompagner la souffrance spirituelle nous ramène à l’idée, intuitivement et plus ou moins confusément ressentie par la plupart des praticiens, que la souffrance spirituelle ne regarde pas que les seuls spécialistes de la religion (théologiens ou ministres du culte) mais qu'il s'agit d'une souffrance tellement liée à la notion même d'être humain - une souffrance qui constitue comme une sorte de convocation, au cœur même de la condition d'homme - qu'elle regarde en réalité chacun, fut-il médecin, psychologue, bénévole ou simple proche du malade. Tenter alors d'accompagner la souffrance spirituelle, c'est d'abord identifier la nature du besoin irrésolu de sens qui se tient au cœur de l'homme. Ce besoin peut être schématiquement de deux ordres :

- Un besoin qu'à défaut de mieux on qualifiera d' " existentiel » et qui conduit l'homme à s’interroger sur le sens de la vie, des épreuves et de la mort, soit dans le regard porté sur la cause, l'origine, c'est-à-dire vers le passé (sens rétrospectif: " d'où ça vient? »), soit dans le regard porté sur l'effet, voire la raison d'être, la finalité, c'est-à-dire vers l'avenir (sens prospectif : «à quoi ça sert ? »). C'est le champ du «pourquoi » : pourquoi ça, pourquoi moi, pourquoi maintenant, pourquoi, pour quoi faire ? Ce besoin est un besoin de savoir et un besoin de repères; il appelle une réponse savante.

- Un besoin relationnel qui consiste en un besoin de se sentir relié, non séparé, proche de corps et d'âme. L'être humain, pour se sentir pleinement vivant, a besoin d'éprouver, de sentir et de ressentir la proximité d'un autre et de se sentir considéré, aimé, porté par cet autre. Chacun a besoin d'une altérité pour se sentir exister. Il ne s'agit plus alors d'une soif de sens entendue comme un savoir ( « je sais ») mais d'une soif de sens entendue comme un senti ( « je sens »). Ce besoin est un besoin de sensation et de ressenti ; il appelle une réponse qui soit présence.

Notons que ces besoins correspondent d'ailleurs précisément à la double étymologie mot religion : religere (relire) et religare (relier). C'est ce second besoin - relationnel - qu'on peut le mieux, me semble-t-il, tenir pour le véritable besoin spirituel, besoin-racine de tout être humain ${ }^{8}$ de se sentir considéré, relié et aimé, et de pouvoir dès lors se sentir pleinement vivant, animé 9 et pas seulement animal. C'est ce besoin spirituel que les soins palliatifs ont aussi vocation à accompagner par une disposition de l'être et un authentique état d'esprit de l'accompagnant.

\section{Spirituel et soin(s)}

COMTE-SPONVILLE André, L'esprit de l'athéisme, introduction à une spiritualité sans Dieu, Albin Michel, 2006.

Correspondant à la souffrance « d'arrière-fond» que le Dr. Marie-Sylvie Richard tient également pour la souffrance spirituelle, in JACQUEMIN Jean-Claude (dir), Manuel de soins palliatifs, Dunod, 2002, p119.

Du latin, anima, âme. 


\section{"Prise en compte du spirituel et nouvelles représentations du soin »}

On mesure ainsi que, ayant vocation à répondre globalement à un besoin et une demande complexes émis par le malade, le soin véritable ne saurait se réduire à une science, à une technique, à une compétence que les impératifs (quantifiés) de performance de la santé publique tendent pourtant à privilégier. Faire est important ! Auprès du malade, chacun a bien quelque chose à faire et mobilise pour ce faire des techniques apprises : le médecin diagnostique et prescrit, l'infirmière pose les actes médicaux, le psychologue interprète, le religieux officie, l'assistante sociale conseille...Même le bénévole mobilise un savoir-faire acquis en formation (entrer, se présenter, écouter, toucher, patienter...). Pourtant, il ne suffit pas de faire pour prendre soin. L'acte seul, posé de manière mécanique, a parfois conduit à une médecine qu'on a pu qualifier de " déshumanisée ", guérisseuse peut-être, mais insuffisamment soignante.

Le soin est donc plus que le faire. Il est la triple association d'un faire, d'un être et d'un au nom de..., la triple conjugaison d'un acte (science), de la raison de cet acte (conscience) et de la manière de cet acte (présence). Le pourquoi (raison, au nom de...) justifie le comment doublement décliné dans le registre matériel (acte) et dans le registre spirituel (manière de l'acte). Le soin véritable, c'est donc la coexistence de ces trois éléments (science, conscience, présence) répondant à une demande et à une confiance de la part du malade ${ }^{10}$.

Il y a donc comme une échelle dans le soin avec un niveau physique (l'acte), un niveau éthique (la raison) et un niveau spirituel (la manière) qui le rend de plus en plus plein. " On peut se tuer à la tâche. Si elle n'est pas imprégnée d'amour, elle est inutile " disait Mère Teresa à la lumière de sa longue expérience de l'accompagnement des mourants de Calcutta. Le geste ne devient donc pleinement soin que dans la manière dont il est habité, et le soin ne devient total que dans ses effets de relation et de réunion, sa dimension spirituelle (religare) ; ou alors il n'est que matière du soin, fragment du soin. Au cœur du soin, se tient donc l'offrande de soi (il y a beaucoup de soi dans le soin) et la rencontre de l'autre. Il est littéralement tentative de reconstruction, de réunification, abolition d'un état de division tant intérieur qu'extérieur. Le soin cherche à rétablir le sain, il vise à la fin de la maladie (en lat. male habitus), littéralement le fait d'être " mal habité ». Le partage de présence, à travers des actes véritablement habités, est donc un des constituants du soin véritable.

Dans cet audacieux congrès consacré à la question spirituelle, rappeler que la manière dont nos actes sont habités est essentiel. Pour répondre en conscience à notre vocation, il nous faut penser autrement le soin - à une personne considérée dans son unité physique et psychique - comme la conjugaison d'un geste attentif (faire) et d'une manière attentionnée de l'accomplir (état d'esprit), au nom de la bienfaisance et de la sollicitude, au nom même de notre humanité (conscience) : une manière d'être dans une manière de faire référée à une éthique. Ainsi pensé, le soin devient l'expression même - et la démonstration - de notre humanité, dans toutes ses dimensions.

Penser autrement permet alors d'agir autrement en étant autrement. Il s'agit de poser des actes autrement, des actes médicaux, psychologiques, sociaux... qui soient aussi des actes de soin, par la qualité de présence et de conscience dont on les investit. Il faut 


\section{"Prise en compte du spirituel et nouvelles représentations du soin »}

bien comprendre que le principal outil de l'accompagnant, c'est lui-même. La réflexion autour de la souffrance spirituelle ne permet pas seulement de disposer de nouveaux outils mieux adaptés pour identifier les besoins des malades et les accompagner. Elle permet d'abord de changer de regard, de référence. Comprendre pour nous-mêmes d'abord ce qu'est le spirituel, c'est ensuite être capable d'accompagner les malades dans ce registre non parce qu'on sait, mais parce qu'on est changé ; non parce qu'on voit autrement mais parce qu'on est devenu autrement. La souffrance des malades nous convoque aussi au dépassement de nous-mêmes, à l'invention de nous-mêmes. Prendre soin de l'autre, c'est d'abord se risquer soi-même dans l'exploration de sa propre dimension spirituelle. Comme Antigone, accompagnant Edipe, le père-frère aveugle et boiteux, banni de sa cité, se risque tout entière sur une voie inconnue, dans la découverte et le jaillissement d'elle-même. Réintroduire sans complexe le spirituel au cœur de nos pratiques palliatives est une attitude essentielle en ce qu'elle nous convoque à notre propre essence, nous transforme et de fait transforme nos actes. Poser des actes palliatifs repose sur une technique. Accompagner en posant des actes palliatifs sollicite notre fréquentation et notre familiarité avec l'essentiel. L'immense perspective des soins palliatifs, c'est de parvenir à placer au chevet de « souffrants globaux " des soignants (au sens large) également globaux. En cela les mourants et leurs accompagnants cheminent ensemble et font "société » au service d'une humanité partagée.

Le soin requiert cependant une patience à l'égard du patient, qui s'accommode mal de l'urgence et de la vision technicienne qui tend à dominer la médecine contemporaine. La responsabilité des acteurs de soins palliatifs, c'est donc aujourd'hui d'oser résister à la tentation de la technicité (le geste inhabité) et à la pression de l'urgence (l'agitationn ${ }^{11}$ ) pour faire émerger une autre conception et une autre pratique du soin. Si nous laissons les soins palliatifs devenir un dispositif essentiellement technique, codifié, fait de protocoles, nous risquons d'en devenir alors les fossoyeurs en les laissant se réduire à une discipline médicale en plus, alors qu'ils sont la quête même du soin et l'expression de l'esprit même de la médecine. Les acteurs des soins palliatifs doivent demeurer des révoltés, des insoumis, des agitateurs (pas des agités). Des consciences alertes, vigilantes, inflexibles. C'est beaucoup plus essentiel et même vital qu'on ne le croit pour notre société. Il nous faut la réanimer en mettant en avant la sensibilité, l'intime et l'implication personnelle, et pas seulement la compétence, la neutralité, la démarche qualité...Il nous faut assumer sans complexe, avec fierté même, cette part de spirituel dans notre engagement et dans nos pratiques. C'est aussi parce qu'ils nous conduisent à une représentation spiritualisée du soin que les soins palliatifs constituent, selon le mot du philosophe Michel Serres au congrès 2005 de la SFAP, une véritable « rupture anthropologique " dont nous sommes ensemble les porteurs. En osant préciser et considérer le spirituel dans le soin, c'est bel et bien l'esprit des origines que nous cherchons à retrouver et à raviver. Il nous faut réécouter avec un cœur presque neuf le chant subtil du mot "soin ». A défaut, nous serions passés à côté de ce soin véritable et total qui est notre commune raison d'être.

Patience et présence, silence et écoute, impuissance et humilité, confiance et doute, tristesse et joie, solidarité et fraternité, être... tous ces mots qui décrivent si bien le quotidien de l'accompagnement sont d'abord, faut-il le rappeler, des mots qui caractérisent fondamentalement toutes les voies spirituelles du monde. En ce sens, accompagner est une attitude très exactement spirituelle. 